\title{
Biotechnology and Microbial Neurochemistry: Biotechnological Implications of the Role of Neuromediators in Microbial Systems
}

\author{
Alexander Vladimirovich Oleskin ${ }^{1 *}$, Ol'ga Gennadievna Zhilenkova ${ }^{2}$ and Ilya Romanovich Vodolazov ${ }^{1}$ \\ ${ }^{1}$ Department of General Ecology, Moscow State University, Russia \\ ${ }^{2}$ Gabrichevsky Research Institute of Epidemiology and Microbiology, Russia
}

Submission: February 14, 2017; Published: March 21, 2017

*Corresponding author: Oleskin AV, Department of General Ecology, School of Biology, Moscow State University, 119991, Vorobiev Hills 1(12), Moscow, Russia, Tel: +79035072258; Email: aoleskin@rambler.ru

\begin{abstract}
This mini-review article is focused on the biotechnological and biomedical implications of the novel area of research called microbial endocrinology and, in particular, its recently developed subfield microbial neurochemistry. Of direct biotechnological relevance are the following facts: (a) microorganisms produce neuroactive substances that are of biomedical and psychiatric importance; traditional selection techniques and genetic engineering can be used to obtain microbial super producers of these chemicals and (b) in microbial systems, many neurochemicals produce specific effects such as growth stimulation and regulation of the planktonic cells- biofilm transition, and, therefore, can potentially be used to promote microbial synthesis of valuable biotechnological products.
\end{abstract}

Keywords: Biotechnology; Microbial endocrinology; Neurochemistry; Neurochemicals; Neuromediators; Biogenic amines; Neuroactive amino acids; Growth stimulators; Biofilms

Abbreviations: CNS: Central Nervous System; DOPA: 2,3-Dihydroxy Penyl Alanine; ENS: Enteric Nervous System; GABA: $\gamma$-aminobutyric acid; GI: Gastro Intestinal; QS: Quorum Sensing

\section{Introduction}

Biotechnology was officially defined by the UN Convention on Biological Diversity as "any technological application that uses biological systems, living organisms, or derivatives thereof, to make or modify products or processes for specific use" [1]. Among microbial products, of special note are neuroactive substances that regulate and modify the functioning of the CNS (the brain). Therefore, they are of considerable importance in clinical, psychiatric, and behavioral terms. Microbial production of neurochemicals is an area of research that straddles the boundary between biotechnology and microbial endocrinology that itself represents a "borderline" interdisciplinary field. It was defined by its proponent Lyte [2] as "the intersection of two seemingly disparate fields, microbiology and neurobiology, and is based on the shared presence of neurochemicals that are exactly the same in host as in the microorganism". Since this review mainly deals with microbial substances that can directly influence impulse transmission between nervous cells across the synaptic cleft, we suggest the term microbial neurochemistry to denote the relevant subfield of microbial endocrinology. The two main aspects of the interaction between microbial neurochemistry and biotechnology are as follows:

a. Microorganisms produce neuroactive substances that are of considerable importance for pharmaceutical biotechnology and

b. In microbial systems, including these used for biotechnological purposes, neurochemicals produce specific effects such as growth stimulation and regulation of the planktonic cells-biofilm transition. These aspects will be discussed in the following sections of this work.

\section{Microbial production of neurochemicals}

Recently, it has been established that various proand eukaryotic microorganisms produce a large number of neurochemicals including biogenic amines such as catecholamines, serotonin, histamine, acetylcholine, and agmatine and amino acids such as glutamic acid, aspartic 
acid, $\gamma$-aminobutyric acid (GABA), taurine, and others [3-11]. For instance, Escherichia coli K-12 synthesizes dopamine, norepinephrine, and serotonin; they are present in the culture liquid during the later culture growth stages at concentrations of $\sim 10-20 \mathrm{nM}$ [4]. Some strains of probiotic lactic-acid bacteria that were tested in $[8,9]$ also produced these amines; this is exemplified by the strain Lactobacillus helveticus 100ash whose culture liquid contained $0.4 \mathrm{mM}$ serotonin. Lactobacilli also formed neuroactive amino acids, particularly GABA and glutamic acid $[8,9]$.

As the aforementioned data indicate, the concentrations of most microbially produced neurochemicals were too low for their sustainable biotechnological production at the industrial level. Nevertheless, efficient selection methods including genetic engineering techniques enable us to obtain overproducers of valuable neuroactive compounds. GABA-producing bacteria that release millimoles of GABA into the culture fluid include Lactobacillus and Lactococcus strains from Italian cheese, Chinese adzuki beans, and fermented cod intestines (reviewed, [10]), as well as a number of lactobacilli and bifidobacteria that were isolated from the GI tract of people in the Central Region of Russia [12,13]. For instance, the strain Bifidobacterium adolescentis 150 released up to $5.6 \mathrm{~g} / \mathrm{l}$, i.e., $\sim 50 \mathrm{mM}$, GABA into the medium [12]. Importantly, GABA is a major neuromediator of the CNS and the enteric nervous system (ENS). It improves sleep, concentration, and attention, exerts a relaxing and pacifying influence on the human brain, stimulates metabolic processes in the brain, promotes creative thinking, helps restore speech and locomotive activity after injuries, and functions as an antioxidant (reviewed, [10]).

In addition to GABA, bacteria can produce large amounts of other neuroactive amino acids that are useful drugs for preventive and therapeutic purposes. The probiotic strain Lactobacillus casei K3III24 was established to produce micromolar concentrations of glutamic acid and taurine $[8,9]$. Notably, taurine improves vision, among other useful therapeutic effects. Apart from catecholamines, E. coli, Bacillus cereus, and lactobacilli produce micromolar amounts of their precursor, 2,3-dihydroxyphenylalanine (DOPA) [4, 6, 8, 9]. Interestingly, DOPA passes the blood--brain barrier and is converted to dopamine and norepinephrine in the brain. The catecholamines are known to regulate important brain processes. They are involved in locomotion, affection, sociable and dominant behavior, as well as aggression. Hence the release of micromolar amounts of DOPA by efficient microbial producers, including probiotics, results in producing a significant effect on human psyche and behavior (see [6]). DOPA is also used to treat problems associated with decreased dopamine concentrations in the brain including Parkinson disease in which the substantia nigra of the brain is dopamine-depleted [14]. Therefore, these results hold much promise with respect to the employment of DOPA in the form of probiotic cultures or dairy products fermented by lactobacilli that serve as DOPA overproducers [10].
Bifidobacteria, another group of probiotics, seem to be much less efficient in producing neurochemicals, based on our recent preliminary data obtained on a yeast extract-containing medium (Oleskin, Zhilenkova, and Vodolazov, unpublished).

As for eukaryotic microorganisms, the yeast Saccharomyces cerevisiae accumulates biogenic amines such as dopamine, norepinephrine, serotonin, and DOPA inside their cells without releasing them into the culture liquid $[6,15]$. This fact has practical implications. Yeast-fermented products, including wine and beer, are expected to produce neurochemical effects only if they are not separated from yeast cells by filtration. Upon digestion, the yeast biomass that is contained in unfiltered ("grey") beverages should supply the GI tract with neuromediators.

\section{Microorganisms specifically respond to exogenous neurochemicals}

Lyte [2] (who coined the term "microbial endocrinology") and other researchers revealed that addition of catecholamines, especially of norepinephrine, drastically stimulates the growth of a wide variety of bacteria [16-23]. Unfortunately, the list of catecholamine-stimulated microorganisms includes a large number of pathogens. They are exemplified by the enterohemorrhagic strain E. coli 07:H157 (EHEC) in which norepinephrine, apart from promoting growth, also stimulates production of Shiga-like toxins and adhesins [23]. Interestingly, norepinephrine, as well as serotonin and histamine, stimulate cell aggregation, the initial stage of biofilm formation, in a nonpathogenic strain of E. coli [4]. However, the development of the non-pathogenic strain is more significantly stimulated by serotonin and especially by histamine than by catecholamines $[4,24]$.

Two mechanisms of biogenic amine-induced microbial growth stimulation have been suggested in the literature (reviewed, [18-22]):

A. Catecholamines chelate iron ions and prevent their binding to proteins (lactoferrin, transferrin, etc.) that are present in complex organic, e.g., serum-containing media; this enables bacteria to take up iron and to synthesize hemecontaining proteins and

B. Catecholamines and plausibly other neuromediators function as analogs of quorum-sensing (QS) pheromones including the catecholamine-like AI-3 pheromone of the QS system of E. coli. For instance, catecholamines stimulate the growth of some strains of lactobacilli and bifidobacteria only on the serum-containing SAPI medium. It seems likely, therefore, that the catecholamines serve as iron chelators in this system. They activate the expression of the feoA and feoB genes that are involved in iron transfer in the strain L. plantarum 90sk [12]. As for eukaryotic microorganisms, biogenic amines, and particularly dopamine, considerably stimulate the proliferation of $S$. cerevisiae cells [15]. However, our preliminary data seem to indicate that they do 
not significantly promote the growth of the fungi Aspergillus acracius and Cunninghamella japonica [25].

\section{Conclusion}

It follows from the above that growth-stimulating neurochemicals can potentially be used for promoting microbial synthesis of valuable products. This is exemplified by the work $[12,13]$ cited above, in which catecholamines were employed to stimulate the growth of lactobacilli and bifidobacteria that produce valuable chemicals including GABA. Catecholamines are also involved in regulating the transition between the existence in the form of planktonic cells and biofilm formation in some microbial species (reviewed, [6]. This can also have important biotechnological implications because many products are more efficiently produced by biofilms than by planktonic microbial cells. Generally speaking, the fact that neuromediators produce drastic growth effects at lower micromolar or submicromolar concentrations provides for the potential commercial viability of biotechnological developments that will be based on these substances. It should be re-emphasized that microorganisms can efficiently produce their own neurochemicals, and maximizing their production also seems to be a sufficiently important goal in terms of medical biotechnology. Overall, the intersection of microbial neurochemistry and biotechnology seems to be a promising research and development field.

\section{References}

1. UN Convention on Biolgical Diversity.

2. Lyte M (2016) Microbial endocrinology in the pathogenesis of infectious disease. Microbiol Spectrum 4(2): doi: 10.1128/microbiolspec.VMBF0021-2015.

3. Tsavkelova EA, Botvinko IV, Kudrin VS, Oleskin AV (2000) Detection of neuromediator amines in microorganisms by high-performance liquid chromatography. Dokl Biochem 372(1-6): 115-117.

4. Shishov VA, Kirovskaya TA, Kudrin VS, Oleskin AV (2009) Amine neuromediators, their precursors, and oxidation products in the culture of Escherichia coli K-12. Prikl Biokhim Mikrobiol 45(5): 494497.

5. Özogul F (2004) Production of biogenic amines by Morganella morganii, Klebsiella pneumonia and Hafnia alvii using a rapid HPLC method. Eur Food Res Technol 219(5): 465-469.

6. Oleskin AV, Shishov VI, Malikina KD (2010) Symbiotic Biofilms and Brain Neurochemistry, Nova Science, Hauppauge (New York), USA.

7. Zhilenkova OG, Shenderov BA, Klodt PM, Kudrin VS, Oleskin AV (2013) Dairy products as a potential source of compounds affecting consumer behavior. Mol Prom (Russian) No 10: 16-19.

8. Oleskin AV, Zhilenkova OG, Shenderov BA, Amerhanova AM, Kudrin VS, Klodt PM (2014) Lactic-acid bacteria supplement fermented dairy products with human behavior-modifying neuroactive compounds. J Pharm Nutrit Sci 4: 199-206.
9. Oleskin AV, Zhilenkova OG, Shenderov BA, Amerhanova AM, Kudrin VS, Klodt PM (2014) Starter cultures of lactobacilli as producers of neuromediators such as biogenic amines and amino acids. Mol Prom (Russian) No 9: 42-43.

10. Oleskin AV, El' Registan GI, Shenderov BA (2016) Role of neuromediators in the functioning of the human microbiota: "business talks" among microorganisms and the microbiota-host dialogue. Microbiology 85(1): 3-25.

11. Oleskin AV, Shenderov BA, Rogovsky VS (2017) Role of neurochemicals in the interaction between the microbiota and the immune and the nervous system of the host organism. Probiotics Antimicrob Proteins In press. doi: 10.1007/s12602-017-9262-1.

12. Yunes RA (2017) Adaptive significance of the bacteria of the genera Lactobacillus and Bifidobacterium for humans. Summary of the Cand Sci (Biol.) Dissertation. RUDN University, Moscow, Russia.

13. Yunes RA, Klimina KM, Emelyanov KV, Zakharevich NZ (2015) Draft genome sequences of Lactobacillus plantarum strain 90sk and Lactobacillus brevis strain 15f: focusing on neurotransmitter genes. Genome Anounc 3(2): e00261-15.

14. Dubynin VA, Kamenskii AA, Sapin MR, Sivoglazov VN (2003) Regulatory systems of the human organism. Drofa, Moscow, Russia .

15. Malikina KD, Shishov VA, Chuvelev DI, Kudrin VS, Oleskin AV (2010) Regulatory role of neuromediator amines in Saccharomyces cerevisiae cells. Prikl Biokhim Mikrobiol 46(6): 672-677.

16. Lyte M (1993) The role of microbial endocrinology in infectious disease. J Endocrinol 137(3): 343-345.

17. Lyte M, Ernst S (1992) Catecholamine induced growth of gram negative bacteria. Life Sci 50(3): 203-212.

18. Lyte M (2010) The microbial organ in the gut as a driver of homeostasis and disease. Med Hypotheses 74(4): 634-638.

19. Lyte M (2011) Probiotics function mechanistically as delivery vehicles for neuroactive compounds: microbial endocrinology in the design and use of probiotics. Bioessays 33(8): 574-581.

20. Lyte M (2013) Microbial endocrinology and nutrition: A perspective on new mechanisms by which diet can influence gut-to braincommunication. Pharma Nutrition 1(1): 35-39.

21. Lyte M (2016) Microbial endocrinology in the pathogenesis of infectious disease. Microbiol Spectr 4(2): VMBF-0021-2015. doi: 10.1128/microbiolspec.VMBF-0021-2015.

22. Freestone PP, Lyte M (2008) Microbial endocrinology: Experimental design issues in the study of interkingdom signaling in infectious disease. Adv Appl Microbiol 64: 75-108.

23. Lyte M, Frank CD, Green BT (1996) Production of an autoinducer of growth by norepinephrine-cultured Escherichia coli 0157:H7. FEMS Microbiol Lett 139(2-3): 155-159.

24. Oleskin AV, Kirovskaya TA, Botvinko IV, Lysak LV (1998) Effect of serotonin (5-hydroxytryptamine) on the growth and differentiation of microorganisms. Mikrobiologiia 67(3): 306-311.

25. Oleskin AV, Vodolazov IR, Zhilenkova OG, Shenderov BA (2016) Dopamine, norepinephrine, and serotonin produce no significant effect on the growth of Lactobacillus acidophila. Jena, International Study Group for Systems Biology (ISGSB) Meeting. 
